\title{
The generalized viscosity explicit rules for a family of strictly pseudo-contractive mappings in a $q$-uniformly smooth Banach space
}

Wongvisarut Khuangsatung ${ }^{1}$ and Pongsakorn Sunthrayuth ${ }^{1 *}$

\section{"Correspondence: \\ pongsakorn_su@rmutt.ac.th 1 Department of Mathematics and Computer Science, Faculty of Science and Technology, Rajamangala University of Technology Thanyaburi (RMUTT) Pathumthani, Thailand}

\begin{abstract}
In this paper, we construct an iterative method by a generalized viscosity explicit rule for a countable family of strictly pseudo-contractive mappings in a q-uniformly smooth Banach space. We prove strong convergence theorems of proposed algorithm under some mild assumption on control conditions. We apply our results to the common fixed point problem of convex combination of family of mappings and zeros of accretive operator in Banach spaces. Furthermore, we also give some numerical examples to support our main results.
\end{abstract}

Keywords: Strict pseudo-contractions; Banach space; Strong convergence; Fixed point problem; Iterative method

\section{Introduction}

In this paper, we assume that $E$ is a real Banach space with dual space $E^{*}$ and $C$ is a nonempty subset of $E$. Let $q>1$ be a real number. The generalized duality mapping $J_{q}: E \rightarrow 2^{E^{*}}$ is defined by

$$
J_{q}(x)=\left\{\bar{x} \in E^{*}:\langle x, \bar{x}\rangle=\|x\|^{q},\|\bar{x}\|=\|x\|^{q-1}\right\},
$$

where $\langle\cdot, \cdot\rangle$ denotes the generalized duality pairing between elements of $E$ and $E^{*}$. In particular, $J_{q}=J_{2}$ is called the normalized duality mapping. If $E$ is smooth, then $J_{q}$ is singlevalued and denoted by $j_{q}$ (see [1]). If $E:=H$ is a real Hilbert space, then $J=I$, where $I$ is the identity mapping. Further, we have the following properties of the generalized duality mapping $J_{q}$ :

- $J_{q}(x)=\|x\|^{q-2} J_{2}(x)$ for all $x \in E$ with $x \neq 0$.

- $J(t x)=t^{q-1} J_{q}(x)$ for all $x \in E$ and $t \geq 0$.

- $J_{q}(-x)=-J_{q}(x)$ for all $x \in E$.

Let $T$ be a self-mapping of $C$. We denote the fixed point set of the mapping $T$ by $F(T)=$ $\{x \in C: x=T x\}$. A mapping $f: C \rightarrow C$ is said to be a contraction if there exists a constant $\rho \in(0,1)$ satisfying

$$
\|f(x)-f(y)\| \leq \rho\|x-y\|, \quad \forall x, y \in C .
$$

(c) The Author(s) 2018. This article is distributed under the terms of the Creative Commons Attribution 4.0 International License (http://creativecommons.org/licenses/by/4.0/), which permits unrestricted use, distribution, and reproduction in any medium, provided you give appropriate credit to the original author(s) and the source, provide a link to the Creative Commons license, and indicate if changes were made. 
We use $\Pi_{C}$ to denote the collection of all contractions from $C$ into itself. Recall that a mapping $T: C \rightarrow C$ is said to be nonexpansive if

$$
\|T x-T y\| \leq\|x-y\|, \quad \forall x, y \in C .
$$

A mapping $T: C \rightarrow C$ is said to be $\lambda$-strict pseudo-contraction if for all $x, y \in C$, there exist $\lambda>0$ and $j_{q}(x-y) \in J_{q}(x-y)$ such that

$$
\left\langle T x-T y, j_{q}(x-y)\right\rangle \leq\|x-y\|^{q}-\lambda\|(I-T) x-(I-T) y\|^{q}, \quad \forall x, y \in C .
$$

It is not hard to show that (1) equivalent to the following inequality:

$$
\left\langle(I-T) x-(I-T) y, j_{q}(x-y)\right\rangle \geq \lambda\|(I-T) x-(I-T) y\|^{q}, \quad \forall x, y \in C .
$$

If $E:=H$ is a Hilbert space, then (1) (and so (2)) is equivalent to the following inequality:

$$
\|T x-T y\|^{2} \leq\|x-y\|^{2}+k\|(I-T) x-(I-T) y\|^{2}, \quad \forall x, y \in C,
$$

where $k=1-2 \lambda<1$. We assume that $k \geq 0$, so that $k \in[0,1)$. Note that the class of strictly pseudo-contractive mappings include the class of nonexpansive mappings as a particular case in Hilbert spaces. Clearly, $T$ is nonexpansive if and only if $T$ is a 0 -strict pseudocontraction. Strict pseudo-contractions were first introduced by Browder and Petryshyn [2] in 1967. They have more powerful applications than nonexpansive mappings do in solving inverse problems (see, e.g., [3]). Therefore it is more interesting to study the theory of iterative methods for strictly pseudo-contractive mappings. Several researchers studied the class of strictly pseudo-contractive mappings in Hilbert and Banach spaces (see, e.g., [4-9] and the references therein).

Now, we give some examples of $\lambda$-strictly pseudo-contractive mappings.

Example 1.1 ([8]) Let $E=\mathbb{R}$ with the usual norm, and let $C=(0, \infty)$. Let $T: C \rightarrow C$ be defined by

$$
T x=\frac{x^{2}}{1+x}, \quad x \in C .
$$

Then, $T$ is a 1-strict pseudo-contraction.

Example 1.2 ([8]) Let $E=\mathbb{R}$ with the usual norm, and let $C=[-1,1]$. Let $T: C \rightarrow C$ be defined by

$$
T x= \begin{cases}x, & x \in[-1,0], \\ x-x^{2}, & x \in[0,1] .\end{cases}
$$

Then, $T$ is a $\lambda$-strict pseudo-contraction with constant $\lambda>0$.

Over the last several years, the implicit midpoint rule (IMR) has become a powerful numerical method for numerically solving time-dependent differential equations (in particular, stiff equations) (see [10-15]) and differential algebraic equations (see [16]). Consider 
the following initial value problem:

$$
x^{\prime}(t)=f(x(t)), \quad x\left(t_{0}\right)=x_{0},
$$

where $f: \mathbb{R}^{M} \rightarrow \mathbb{R}^{M}$ is a continuous function. The IMR is an implicit method given by the following finite difference scheme [17]:

$$
\left\{\begin{array}{l}
y_{0}=x_{0} \\
y_{n+1}=y_{n}+h f\left(\frac{y_{n}+y_{n+1}}{2}\right), \quad n \geq 0
\end{array}\right.
$$

where $h>0$ is a time step. It is known that if $f: \mathbb{R}^{M} \rightarrow \mathbb{R}^{M}$ is Lipschitz continuous and sufficiently smooth, then the sequence $\left\{y_{n}\right\}$ converges to the exact solution of (4) as $h \rightarrow 0$ uniformly over $t \in\left[t_{0}, t^{*}\right]$ for any fixed $t^{*}>0$. If the function $f$ is written as $f(x)=x-g(x)$, then (5) becomes

$$
\left\{\begin{array}{l}
y_{0}=x_{0}, \\
y_{n+1}=y_{n}+h\left[\frac{y_{n}+y_{n+1}}{2}-g\left(\frac{y_{n}+y_{n+1}}{2}\right)\right], \quad n \geq 0,
\end{array}\right.
$$

and the critical points of (4) are the fixed points of the problem $x=g(x)$.

Based on IMR (5), Alghamdi et al. [18] introduced the following two algorithms for the solution of the fixed point problem $x=T x$, where $T$ is a nonexpansive mapping in a Hilbert space $H$ :

$$
\begin{aligned}
& x_{n+1}=x_{n}-t_{n}\left[\frac{x_{n}+x_{n+1}}{2}-T\left(\frac{x_{n}+x_{n+1}}{2}\right)\right], \quad n \geq 0, \\
& x_{n+1}=\left(1-t_{n}\right) x_{n}+t_{n} T\left(\frac{x_{n}+x_{n+1}}{2}\right), \quad n \geq 0,
\end{aligned}
$$

for $x_{0} \in H$, with $\left\{t_{n}\right\}_{n=1}^{\infty} \subset(0,1)$. They proved that these two schemes converge weakly to a point in $F(T)$.

To obtain strong convergence, $\mathrm{Xu}$ et al. [19] applied the viscosity approximation method introduced by Moudafi [20] to the IMR for a nonexpansive mapping $T$ and proposed the following viscosity implicit midpoint rule in Hilbert spaces $H$ as follows:

$$
x_{n+1}=\alpha_{n} f\left(x_{n}\right)+\left(1-\alpha_{n}\right) T\left(\frac{x_{n}+x_{n+1}}{2}\right), \quad n \geq 1,
$$

where $\left\{\alpha_{n}\right\}$ is a real control condition in $(0,1)$. They also proved that the sequence $\left\{x_{n}\right\}$ generated by (9) converges strongly to a point $x^{*} \in F(T)$, which solves the variational inequality

$$
\left\langle(f-I) x^{*}, z-x^{*}\right\rangle \leq 0, \quad z \in F(T) .
$$

Later, Ke and Ma [21] improved the viscosity implicit midpoint rule by replacing the midpoint by any point of the interval $\left[x_{n}, x_{n+1}\right]$. They introduced the so-called generalized viscosity implicit rules to approximating the fixed point of a nonexpansive mapping $T$ in 
Hilbert spaces $H$ as follows:

$$
x_{n+1}=\alpha_{n} f\left(x_{n}\right)+\left(1-\alpha_{n}\right) T\left(s_{n} x_{n}+\left(1-s_{n}\right) x_{n+1}\right), \quad n \geq 1 .
$$

They also proved that the sequence $\left\{x_{n}\right\}$ generated by (11) converges strongly to a point $x^{*} \in F(T)$ that solves the variational inequality (10).

In numerical analysis, it is clear that the computation by the IMR is not an easy work in practice. Because the IMR need to compute at every time steps, it can be much harder to implement. To overcome this difficulty, for solving (4), we consider the helpful method, the so-called explicit midpoint method (EMR), given by the following finite difference scheme $[22,23]$ :

$$
\left\{\begin{array}{l}
y_{0}=x_{0}, \\
\bar{y}_{n+1}=y_{n}+h f\left(y_{n}\right), \\
y_{n+1}=y_{n}+h f\left(\frac{y_{n}+\bar{y}_{n+1}}{2}\right), \quad n \geq 0 .
\end{array}\right.
$$

Note that the EMR (12) calculates the system status at a future time from the currently known system status, whereas IMR (5) calculates the system status involving both the current state of the system and the later one (see $[23,24])$.

In 2017, Marino et al. [25] combined the generalized viscosity implicit midpoint rules (11) with the EMR (12) for a quasi-nonexpansive mapping $T$ and introduced the following so-called generalized viscosity explicit midpoint rule in Hilbert spaces $H$ as follows:

$$
\left\{\begin{array}{l}
\bar{x}_{n+1}=\beta_{n} x_{n}+\left(1-\beta_{n}\right) T x_{n} \\
x_{n+1}=\alpha_{n} f\left(x_{n}\right)+\left(1-\alpha_{n}\right) T\left(s_{n} x_{n}+\left(1-s_{n}\right) \bar{x}_{n+1}\right), \quad n \geq 1 .
\end{array}\right.
$$

They also showed that, under certain assumptions imposed on the parameters, the sequence $\left\{x_{n}\right\}$ generated by (13) converges strongly to a point $x^{*} \in F(T)$, which solves the variational inequality (10).

The above results naturally bring us to the following questions.

Question 1 Can we extend the generalized viscosity explicit midpoint rule (13) to higher spaces other than Hilbert spaces? Such as a 2-uniformly smooth Banach space or, more generally, in a $q$-uniformly smooth Banach space.

Question 2 Can we obtain a strong convergence result of generalized viscosity explicit midpoint rule (13) for finding the set of common fixed points of a family of mappings? Such as a countable family of strict pseudo-contractions.

The purpose of this paper is to give some affirmative answers to the questions raised. We introduce an iterative algorithm for finding the set of common fixed points of a countable family of strict pseudo-contractions by a generalized viscosity explicit rule in a $q$ uniformly smooth Banach space. We prove the strong convergence of the proposed algorithm under some mild assumption on control conditions. We apply our results to the common fixed point problem of a convex combination of a family of mappings and zeros of an accretive operator in Banach spaces. Furthermore, we also give some numerical examples to support our main results. 


\section{Preliminaries}

Let $E$ be a real Banach space with norm $\|\cdot\|$ and dual space $E^{*}$ of $E$. The symbol $\left\langle x, x^{*}\right\rangle$ denotes the pairing between $E$ and $E^{*}$, that is, $\left\langle x, x^{*}\right\rangle=x^{*}(x)$, the value of $x^{*}$ at $x$. The modulus of convexity of $E$ is the function $\delta:(0,2] \rightarrow[0,1]$ defined by

$$
\delta(\epsilon)=\inf \left\{1-\frac{\|x+y\|}{2}: x, y \in E,\|x\|=\|y\|=1,\|x-y\| \geq \epsilon\right\} .
$$

A Banach space $E$ is said to be uniformly convex if $\delta_{E}(\epsilon)>0$ for all $\epsilon \in(0,2]$. For $p>1$, we say that $E$ is said to be $p$-uniformly convex if there is $c_{p}>0$ such that $\delta_{E}(\epsilon) \geq c_{p} \epsilon^{p}$ for all $\epsilon \in(0,2]$.

The modulus of smoothness of $E$ is the function $\rho_{E}: \mathbb{R}^{+}:=[0, \infty) \rightarrow \mathbb{R}^{+}$defined by

$$
\rho_{E}(\tau)=\sup \left\{\frac{\|x+\tau y\|+\|x-\tau y\|}{2}-1:\|x\|,\|y\| \leq 1\right\} .
$$

A Banach space $E$ is said to be uniformly smooth if $\frac{\rho_{E}(\tau)}{\tau} \rightarrow 0$ as $\tau \rightarrow 0$. For $q>1$, a Banach space $E$ is said to be q-uniformly smooth if there exists $c_{q}>0$ such that $\rho_{E}(\tau) \leq c_{q} \tau^{q}$ for all $\tau>0$. If $E$ is $q$-uniformly smooth, then $q \leq 2$, and $E$ is also uniformly smooth. Further, $E$ is $p$-uniformly convex ( $q$-uniformly smooth) if and only if $E^{*}$ is $q$-uniformly smooth ( $p$-uniformly convex), where $p \geq 2$ and $1<q \leq 2$ satisfy $\frac{1}{p}+\frac{1}{q}=1$. It is well known that Hilbert spaces $L_{p}$ and $l_{p}(p>1)$ are uniformly smooth (see [26]). More precisely, the spaces $L_{p}$ and $l_{p}$ are $\min \{p, 2\}$-uniformly smooth for every $p>1$.

Definition 2.1 Let $C$ a be nonempty closed convex subsets of $E$, and let $Q$ be a mapping of $E$ onto $C$. Then $Q$ is said to be:

- sunny if $Q(Q x+t(x-Q x))=Q x$ for all $x \in C$ and $t \geq 0$.

- retraction if $Q x=x$ for all $x \in C$.

- a sunny nonexpansive retraction if $Q$ is sunny, nonexpansive, and a retraction from $E$ onto $C$.

It is known that if $E:=H$ is a real Hilbert space, then a sunny nonexpansive retraction $Q$ coincides with the metric projection from $E$ onto $C$. Moreover, if $E$ is uniformly smooth and $T$ is a nonexpansive mapping of $C$ into itself with $F(T) \neq \emptyset$, then $F(T)$ is a sunny nonexpansive retraction from $E$ onto $C$ (see [27]). We know that in a uniformly smooth Banach space, a retraction $Q: C \rightarrow E$ is sunny and nonexpansive if and only if $\left\langle x-Q x, j_{q}(y-\right.$ $Q x)\rangle \leq 0$ for all $x \in E$ and $y \in C$ (see [28]).

Lemma 2.2 ([29]) Let C be a nonempty closed convex subset of a uniformly smooth Banach space $E$. Let $S: C \rightarrow C$ be a nonexpansive self-mapping such that $F(S) \neq \emptyset$ and $f \in \Pi_{C}$. Let $\left\{z_{t}\right\}$ be the net sequence defined by

$$
z_{t}=t f\left(z_{t}\right)+(1-t) S z_{t}, \quad t \in(0,1) .
$$

Then:

(i) $\left\{x_{t}\right\}$ converges strongly as $t \rightarrow 0$ to a point $Q(f) \in F(S)$, which solves the variational inequality

$$
\left\langle(I-f) Q(f), j_{q}(Q(f)-z)\right\rangle \leq 0, \quad z \in F(S) .
$$


(ii) Suppose that $\left\{x_{n}\right\}$ is a bounded sequence such that $\lim _{n \rightarrow \infty}\left\|x_{n}-S x_{n}\right\|=0$. If $Q(f):=\lim _{t \rightarrow 0} x_{t}$ exists, then

$$
\limsup _{n \rightarrow \infty}\left\langle(f-I) Q(f), j_{q}\left(x_{n}-Q(f)\right)\right\rangle \leq 0
$$

Lemma 2.3 ([30]) Let $C$ be a nonempty closed convex subset of a real q-uniformly smooth Banach space E. Let $T: C \rightarrow C$ be a $\lambda$-strict pseudo-contraction. For all $x \in C$, we define $T_{\theta} x:=(1-\theta) x+\theta T x$. Then, as $\theta \in(0, \delta], \delta=\min \left\{1,\left(\frac{q \lambda}{\kappa_{q}}\right)^{\frac{1}{q-1}}\right\}$, where $\kappa_{q}$ is the q-uniform smoothness constant, and $T_{\theta}: C \rightarrow C$ is nonexpansive such that $F\left(T_{\theta}\right)=F(T)$.

Using the concept of subdifferentials, we have the following inequality.

Lemma 2.4 ([31]) Let $q>1$, and let $E$ be a real normed space with the generalized duality mapping $J_{q}$. Then, for any $x, y \in E$, we have

$$
\|x+y\|^{q} \leq\|x\|^{q}+q\left\langle y, j_{q}(x+y)\right\rangle
$$

where $j_{q}(x+y) \in J_{q}(x+y)$.

Lemma 2.5 ([32]) Let $p>1$ and $r>0$ be two fixed real numbers, and let E be a uniformly convex Banach space. Then, for all $x, y \in B_{r}$ and $t \in[0,1]$,

$$
\|t x+(1-t) y\|^{p} \leq t\|x\|^{p}+(1-t)\|y\|^{p}-t(1-t) c\|x-y\|^{p},
$$

where $c>0$.

Lemma 2.6 ([33]) Suppose that $q>1$. Then

$$
a b \leq \frac{1}{q} a^{q}+\left(\frac{q-1}{q}\right) b^{\frac{q}{q-1}}
$$

for positive real numbers $a, b$.

Lemma 2.7 ([34]) Let $\left\{a_{n}\right\}$ be a sequence of nonnegative real numbers, $\left\{\gamma_{n}\right\}$ be a sequence of $(0,1)$ with $\sum_{n=1}^{\infty} \gamma_{n}=\infty,\left\{c_{n}\right\}$ be a sequence of nonnegative real number with $\sum_{n=1}^{\infty} c_{n}<\infty$, and let $\left\{b_{n}\right\}$ be a sequence of real numbers with $\limsup _{n \rightarrow \infty} b_{n} \leq 0$. Suppose that

$$
a_{n+1}=\left(1-\gamma_{n}\right) a_{n}+\gamma_{n} b_{n}+c_{n}
$$

for all $n \in \mathbb{N}$. Then, $\lim _{n \rightarrow \infty} a_{n}=0$.

Lemma 2.8 ([35]) Let $\left\{s_{n}\right\}$ be sequences of real numbers such that there exists a subsequence $\left\{n_{i}\right\}$ of $\{n\}$ such that $s_{n_{i}}<s_{n_{i}+1}$ for all $i \in \mathbb{N}$. Then there exists an increasing sequence $\left\{m_{k}\right\} \subset \mathbb{N}$ such that $\lim _{k \rightarrow \infty} m_{k}=\infty$ and the following properties are satisfied by all sufficiently large numbers $k \in \mathbb{N}$ :

$$
s_{m_{k}} \leq s_{m_{k}+1} \text { and } s_{k} \leq s_{m_{k}+1}
$$

In fact, $m_{k}:=\max \left\{j \leq k: s_{j} \leq s_{j+1}\right\}$. 
Definition 2.9 ([34]) Let $C$ be a nonempty closed convex subset of a real Banach space $E$. Let $\left\{T_{n}\right\}_{n=1}^{\infty}$ be a family of mappings of $C$ into itself. We say that $\left\{T_{n}\right\}_{n=1}^{\infty}$ satisfies the $A K T T$ condition if

$$
\sum_{n=1}^{\infty} \sup _{w \in C}\left\|T_{n+1} w-T_{n} w\right\|<\infty .
$$

Lemma 2.10 ([34]) Let $C$ be a nonempty closed convex subset of a real Banach space $E$. Suppose that $\left\{T_{n}\right\}_{n=1}^{\infty}$ satisfies the AKTT-condition. Then, for each $x \in C,\left\{T_{n} x\right\}$ converges strongly to some point of $C$. Moreover, let $T$ be the mapping of $C$ into itself defined by $T x=$ $\lim _{n \rightarrow \infty} T_{n} x$ for all $x \in C$. Then, $\lim _{n \rightarrow \infty} \sup _{w \in C}\left\|T w-T_{n} w\right\|=0$.

In the following, we will write that $\left(\left\{T_{n}\right\}, T\right)$ satisfies the $A K T T$-condition if $\left\{T_{n}\right\}$ satisfies the $A K T T$-condition and $T$ is defined by Lemma 2.10 with $F(T)=\bigcap_{n=1}^{\infty} F\left(T_{n}\right)$.

\section{Main results}

Theorem 3.1 Let $C$ be a nonempty closed convex subset of a real uniformly convex and q-uniformly smooth Banach space E. Let $f \in \Pi_{C}$ with coefficient $\rho \in(0,1)$, and let $\left\{T_{n}\right\}_{n=1}^{\infty}$ : $C \rightarrow C$ be a family of $\lambda$-strict pseudo-contractions such that $\Omega:=\bigcap_{n=1}^{\infty} F\left(T_{n}\right) \neq \emptyset$. For all

$x \in C$, define the mapping $S_{n} x=\left(1-\theta_{n}\right) x+\theta_{n} T_{n} x$, where $0<\theta_{n} \leq \delta, \delta=\min \left\{1,\left(\frac{q \lambda}{\kappa_{q}}\right)^{\frac{1}{q-1}}\right\}$, and $\sum_{n=1}^{\infty}\left|\theta_{n+1}-\theta_{n}\right|<\infty$. For given $x_{1} \in C$, let $\left\{x_{n}\right\}$ be a sequence generated by

$$
\left\{\begin{array}{l}
\bar{x}_{n+1}=\beta_{n} x_{n}+\left(1-\beta_{n}\right) S_{n} x_{n}, \\
x_{n+1}=\alpha_{n} f\left(x_{n}\right)+\left(1-\alpha_{n}\right) S_{n}\left(t_{n} x_{n}+\left(1-t_{n}\right) \bar{x}_{n+1}\right), \quad n \geq 1,
\end{array}\right.
$$

where $\left\{\alpha_{n}\right\},\left\{\beta_{n}\right\}$, and $\left\{t_{n}\right\}$ are sequences in $(0,1)$ satisfying the following conditions:

(C1) $\lim _{n \rightarrow \infty} \alpha_{n}=0, \sum_{n=1}^{\infty} \alpha_{n}=\infty$;

(C2) $\liminf _{n \rightarrow \infty} \beta_{n}\left(1-\beta_{n}\right)\left(1-t_{n}\right)>0$.

Suppose in addition that $\left(\left\{T_{n}\right\}_{n=1}^{\infty}, T\right)$ satisfies the AKTT-condition. Then, $\left\{x_{n}\right\}$ defined by (16) converges strongly to $x^{*}=Q(f) \in \Omega$, which solves the variational inequality

$$
\left\langle(I-f) Q(f), j_{q}(Q(f)-z)\right\rangle \leq 0, \quad z \in \Omega
$$

where $Q$ is a sunny nonexpansive retraction of $C$ onto $\Omega$.

Proof First, we show that $\left\{x_{n}\right\}$ is bounded. From Lemma 2.3 we have that $S_{n}$ is nonexpansive such that $F\left(S_{n}\right)=F\left(T_{n}\right)$ for all $n \geq 1$. Put $z_{n}:=t_{n} x_{n}+\left(1-t_{n}\right) \bar{x}_{n+1}$. For each $z \in \Omega:=\bigcap_{n=1}^{\infty} F\left(T_{n}\right)$, we have

$$
\begin{aligned}
\left\|z_{n}-z\right\| & =\left\|t_{n}\left(x_{n}-z\right)+\left(1-t_{n}\right)\left(\bar{x}_{n+1}-z\right)\right\| \\
& \leq t_{n}\left\|x_{n}-z\right\|+\left(1-t_{n}\right)\left\|\bar{x}_{n+1}-z\right\| \\
& \leq t_{n}\left\|x_{n}-z\right\|+\left(1-t_{n}\right)\left(\beta_{n}\left\|x_{n}-z\right\|+\left(1-\beta_{n}\right)\left\|S_{n} x_{n}-z\right\|\right) \\
& \leq t_{n}\left\|x_{n}-z\right\|+\left(1-t_{n}\right) \beta_{n}\left\|x_{n}-z\right\|+\left(1-t_{n}\right)\left(1-\beta_{n}\right)\left\|x_{n}-z\right\| \\
& =\left\|x_{n}-z\right\| .
\end{aligned}
$$


It follows that

$$
\begin{aligned}
\left\|x_{n+1}-z\right\| & =\left\|\alpha_{n} f\left(x_{n}\right)+\left(1-\alpha_{n}\right) S_{n} z_{n}-z\right\| \\
& =\left\|\alpha_{n}\left(f\left(x_{n}\right)-f(z)\right)+\alpha_{n}(f(z)-z)+\left(1-\alpha_{n}\right)\left(S_{n} z_{n}-z\right)\right\| \\
& \leq \alpha_{n}\left\|f\left(x_{n}\right)-f(z)\right\|+\alpha_{n}\|f(z)-z\|+\left(1-\alpha_{n}\right)\left\|S_{n} z_{n}-z\right\| \\
& \leq\left(1-(1-\rho) \alpha_{n}\right)\left\|x_{n}-z\right\|+(1-\rho) \alpha_{n} \frac{\|f(z)-z\|}{1-\rho} \\
& \leq \max \left\{\left\|x_{n}-z\right\|, \frac{\|f(z)-z\|}{1-\rho}\right\} .
\end{aligned}
$$

By induction we have

$$
\left\|x_{n}-z\right\| \leq \max \left\{\left\|x_{1}-z\right\|, \frac{\|f(z)-z\|}{1-\rho}\right\}, \quad n \geq 1
$$

Hence $\left\{x_{n}\right\}$ is bounded. Consequently, we deduce immediately that $\left\{f\left(x_{n}\right)\right\}$ and $\left\{S_{n}\left(t_{n} x_{n}+\right.\right.$ $\left.\left.\left(1-t_{n}\right) \bar{x}_{n+1}\right)\right\}$ are bonded. Let $x^{*}=Q(f)$. By the convexity of $\|\cdot\|^{q}$ and Lemma 2.5 we have

$$
\begin{aligned}
\left\|S_{n} z_{n}-x^{*}\right\|^{q} \leq & \left\|z_{n}-x^{*}\right\|^{q} \\
& =\left\|t_{n}\left(x_{n}-x^{*}\right)+\left(1-t_{n}\right)\left(\bar{x}_{n+1}-x^{*}\right)\right\|^{q} \\
\leq & t_{n}\left\|x_{n}-x^{*}\right\|^{q}+\left(1-t_{n}\right)\left\|\bar{x}_{n+1}-x^{*}\right\|^{q} \\
& =t_{n}\left\|x_{n}-x^{*}\right\|^{q}+\left(1-t_{n}\right)\left\|\beta_{n}\left(x_{n}-x^{*}\right)+\left(1-\beta_{n}\right)\left(S_{n} x_{n}-x^{*}\right)\right\|^{q} \\
\leq & t_{n}\left\|x_{n}-x^{*}\right\|^{q}+\left(1-t_{n}\right)\left[\beta_{n}\left\|x_{n}-x^{*}\right\|^{q}+\left(1-\beta_{n}\right)\left\|S_{n} x_{n}-x^{*}\right\|^{q}\right. \\
& \left.\quad-\beta_{n}\left(1-\beta_{n}\right) c\left\|x_{n}-S_{n} x_{n}\right\|^{q}\right] \\
\leq & \left\|x_{n}-x^{*}\right\|^{q}-\beta_{n}\left(1-\beta_{n}\right)\left(1-t_{n}\right) c\left\|x_{n}-S_{n} x_{n}\right\|^{q} .
\end{aligned}
$$

It follows from Lemma 2.4 and (19) that

$$
\begin{aligned}
&\left\|x_{n+1}-x^{*}\right\|^{q} \\
&=\left\|\alpha_{n}\left(f\left(x_{n}\right)-x^{*}\right)+\left(1-\alpha_{n}\right)\left(S_{n} z_{n}-x^{*}\right)\right\|^{q} \\
&=\left\|\alpha_{n}\left(f\left(x_{n}\right)-f\left(x^{*}\right)\right)+\alpha_{n}\left(f\left(x^{*}\right)-x^{*}\right)+\left(1-\alpha_{n}\right)\left(S_{n} z_{n}-x^{*}\right)\right\|^{q} \\
& \leq\left\|\alpha_{n}\left(f\left(x_{n}\right)-f\left(x^{*}\right)\right)+\left(1-\alpha_{n}\right)\left(S_{n} z_{n}-x^{*}\right)\right\|^{q}+q \alpha_{n}\left\langle f\left(x^{*}\right)-x^{*}, j_{q}\left(x_{n+1}-x^{*}\right)\right\rangle \\
& \leq \alpha_{n}\left\|f\left(x_{n}\right)-f\left(x^{*}\right)\right\|^{q}+\left(1-\alpha_{n}\right)\left\|S_{n} z_{n}-x^{*}\right\|^{q}+q \alpha_{n}\left\langle f\left(x^{*}\right)-x^{*}, j_{q}\left(x_{n+1}-x^{*}\right)\right\rangle \\
& \leq \alpha_{n}\left\|f\left(x_{n}\right)-f\left(x^{*}\right)\right\|^{q}+\left(1-\alpha_{n}\right)\left[\left\|x_{n}-x^{*}\right\|^{q}-\beta_{n}\left(1-\beta_{n}\right)\left(1-t_{n}\right) c\left\|x_{n}-S_{n} x_{n}\right\|^{q}\right] \\
&+q \alpha_{n}\left\langle f\left(x^{*}\right)-x^{*}, j_{q}\left(x_{n+1}-x^{*}\right)\right\rangle \\
& \leq\left(1-(1-\rho) \alpha_{n}\right)\left\|x_{n}-x^{*}\right\|^{q}-\left(1-\alpha_{n}\right) \beta_{n}\left(1-\beta_{n}\right)\left(1-t_{n}\right) c\left\|x_{n}-S_{n} x_{n}\right\|^{q} \\
&+q \alpha_{n}\left\langle f\left(x^{*}\right)-x^{*}, j_{q}\left(x_{n+1}-x^{*}\right)\right\rangle .
\end{aligned}
$$

The rest of the proof will be divided into two cases: 
Case 1. Suppose that there exists $n_{0} \in \mathbb{N}$ such that $\left\{\left\|x_{n}-x^{*}\right\|\right\}_{n=n_{0}}^{\infty}$ is nonincreasing. This implies that $\left\{\left\|x_{n}-x^{*}\right\|\right\}_{n=1}^{\infty}$ is convergent. From (20) we see that

$$
\left(1-\alpha_{n}\right) \beta_{n}\left(1-\beta_{n}\right)\left(1-s_{n}\right) c\left\|x_{n}-S_{n} x_{n}\right\|^{q} \leq\left\|x_{n}-x^{*}\right\|^{q}-\left\|x_{n+1}-x^{*}\right\|^{q}+\alpha_{n} M,
$$

where $c>0$ and $M=\sup _{n \geq 1}\left\{q\left\|f\left(x^{*}\right)-x^{*}\right\|\left\|x_{n+1}-x^{*}\right\|^{q-1},(1-\rho)\left\|x_{n}-x^{*}\right\|^{q}\right\}<\infty$. From $(C 1)$ and $(C 2)$ we get that

$$
\lim _{n \rightarrow \infty}\left\|x_{n}-S_{n} x_{n}\right\|=0
$$

We observe that

$$
\begin{aligned}
& \sup _{x \in\left\{x_{n}\right\}}\left\|S_{n+1} x-S_{n} x\right\| \\
& \quad=\sup _{x \in\left\{x_{n}\right\}}\left\|\left(1-\theta_{n+1}\right) x+\theta_{n+1} T_{n+1} x-\left(1-\theta_{n}\right) x-\theta_{n} T_{n} x\right\| \\
& \quad \leq\left|\theta_{n+1}-\theta_{n}\right| \sup _{x \in\left\{x_{n}\right\}}\|x\|+\theta_{n+1} \sup _{x \in\left\{x_{n}\right\}}\left\|T_{n+1} x-T_{n} x\right\|+\left|\theta_{n+1}-\theta_{n}\right| \sup _{x \in\left\{x_{n}\right\}}\left\|T_{n} x\right\| \\
& \quad \leq\left|\theta_{n+1}-\theta_{n}\right|\left(\sup _{x \in\left\{x_{n}\right\}}\|x\|+\sup _{x \in\left\{x_{n}\right\}}\left\|T_{n} x\right\|\right)+\sup _{x \in\left\{x_{n}\right\}}\left\|T_{n+1} x-T_{n} x\right\| .
\end{aligned}
$$

Since $\left\{T_{n}\right\}_{n=1}^{\infty}$ satisfies the $A K T T$-condition and $\sum_{n=1}^{\infty}\left|\theta_{n+1}-\theta_{n}\right|<\infty$, we have

$$
\sum_{n=1}^{\infty} \sup _{x \in\left\{x_{n}\right\}}\left\|S_{n+1} x-S_{n} x\right\|<\infty,
$$

that is, $\left\{S_{n}\right\}_{n=1}^{\infty}$ satisfies the $A K T T$-condition. From this we can define the nonexpansive mapping $S: C \rightarrow C$ by $S x=\lim _{n \rightarrow \infty} S_{n} x$ for all $x \in C$. Since $\left\{\theta_{n}\right\}$ is bounded, there exists a subsequence $\left\{\theta_{n_{i}}\right\}$ of $\left\{\theta_{n}\right\}$ such that $\theta_{n_{i}} \rightarrow \theta$ as $i \rightarrow \infty$. It follows that

$$
S x=\lim _{i \rightarrow \infty} S_{n_{i}} x=\lim _{i \rightarrow \infty}\left[\left(1-\theta_{n_{i}}\right) x+\theta_{n_{i}} T_{n_{i}} x\right]=(1-\theta) x+\theta T x, \quad x \in C .
$$

This shows that $F(S)=F(T)=\bigcap_{n=1}^{\infty} F\left(T_{n}\right):=\Omega$. By (21) and Lemma 2.10 we have

$$
\begin{aligned}
\left\|x_{n}-S x_{n}\right\| & \leq\left\|x_{n}-S_{n} x_{n}\right\|+\left\|S_{n} x_{n}-S x_{n}\right\| \\
& \leq\left\|x_{n}-S_{n} x_{n}\right\|+\sup _{x \in\left\{x_{n}\right\}}\left\|S_{n} x-S x\right\| \rightarrow 0 \quad \text { as } n \rightarrow \infty .
\end{aligned}
$$

Let $\left\{z_{t}\right\}$ be a sequence defined by

$$
z_{t}=f\left(z_{t}\right)+(1-t) S z_{t}, \quad t \in(0,1)
$$

From Lemma 2.2(i) we know that $\left\{x_{t}\right\}$ converges strongly to $x^{*}=Q(f)$, which solves the variational inequalities

$$
\left\langle(I-f) Q(f), j_{q}(Q(f)-z)\right\rangle \leq 0, \quad z \in \Omega .
$$


Moreover, we obtain that

$$
\limsup _{n \rightarrow \infty}\left\langle f\left(x^{*}\right)-x^{*}, j_{q}\left(x_{n}-x^{*}\right)\right\rangle \leq 0
$$

Note that

$$
\begin{aligned}
\left\|S_{n} z_{n}-x_{n}\right\| & \leq\left\|S_{n} z_{n}-S_{n} x_{n}\right\|+\left\|S_{n} x_{n}-x_{n}\right\| \\
& \leq\left\|z_{n}-x_{n}\right\|+\left\|S_{n} x_{n}-x_{n}\right\| \\
& =\left(1-s_{n}\right)\left(1-\beta_{n}\right)\left\|S_{n} x_{n}-x_{n}\right\|+\left\|S_{n} x_{n}-x_{n}\right\| \\
& \leq 2\left\|x_{n}-S_{n} x_{n}\right\| .
\end{aligned}
$$

From (21), we get that

$$
\lim _{n \rightarrow \infty}\left\|S_{n} z_{n}-x_{n}\right\|=0
$$

It follows that

$$
\begin{aligned}
& \left\|x_{n+1}-x_{n}\right\| \\
& \quad \leq\left\|\alpha_{n}\left(f\left(x_{n}\right)-x_{n}\right)+\left(1-\alpha_{n}\right)\left(S_{n} z_{n}-x_{n}\right)\right\| \\
& \quad \leq \alpha_{n}\left\|f\left(x_{n}\right)-x_{n}\right\|+\left(1-\alpha_{n}\right)\left\|S_{n} z_{n}-x_{n}\right\| \rightarrow 0 \text { as } n \rightarrow \infty .
\end{aligned}
$$

We also have

$$
\limsup _{n \rightarrow \infty}\left\langle f\left(x^{*}\right)-x^{*}, j_{q}\left(x_{n+1}-x^{*}\right)\right\rangle \leq 0
$$

Again from (20), we have

$$
\begin{aligned}
& \left\|x_{n+1}-x^{*}\right\|^{q} \\
& \quad \leq\left(1-(1-\rho) \alpha_{n}\right)\left\|x_{n}-x^{*}\right\|^{q}+q \alpha_{n}\left\langle f\left(x^{*}\right)-x^{*}, j_{q}\left(x_{n+1}-x^{*}\right)\right\rangle .
\end{aligned}
$$

Apply Lemma 2.7 and (26) to (27), we obtain that $x_{n} \rightarrow x^{*}$ as $n \rightarrow \infty$.

Case 2. There exists a subsequence $\left\{n_{i}\right\}$ of $\{n\}$ such that

$$
\left\|x_{n_{i}}-x^{*}\right\| \leq\left\|x_{n_{i+1}}-x^{*}\right\|
$$

for all $i \in \mathbb{N}$. By Lemma 2.8, there exists a nondecreasing sequence $\left\{m_{k}\right\} \subset \mathbb{N}$ such that $m_{k} \rightarrow \infty$ as $k \rightarrow \infty$ and

$$
\left\|x_{m_{k}}-x^{*}\right\| \leq\left\|x_{m_{k}+1}-x^{*}\right\| \quad \text { and } \quad\left\|x_{k}-x^{*}\right\| \leq\left\|x_{m_{k}+1}-x^{*}\right\|
$$

for all $k \in \mathbb{N}$. From (20) we have

$$
\begin{aligned}
& \left(1-\alpha_{m_{k}}\right) \beta_{m_{k}}\left(1-\beta_{m_{k}}\right)\left(1-s_{m_{k}}\right) c\left\|x_{m_{k}}-S_{m_{k}} x_{m_{k}}\right\|^{q} \\
& \quad \leq\left\|x_{m_{k}}-x^{*}\right\|^{q}-\left\|x_{m_{k}+1}-x^{*}\right\|^{q}+\alpha_{m_{k}} M \\
& \quad \leq \alpha_{m_{k}} M
\end{aligned}
$$


where $c>0$ and $M<\infty$. This implies by $(C 1)$ and $(C 2)$ that

$$
\left\|x_{m_{k}}-S_{m_{k}} x_{m_{k}}\right\| \rightarrow 0 \quad \text { as } k \rightarrow \infty
$$

Since

$$
\begin{aligned}
& \sup _{x \in\left\{x_{m_{k}}\right\}}\left\|S_{m_{k}+1} x-S_{m_{k}} x\right\| \\
& =\sup _{x \in\left\{x_{m_{k}}\right\}}\left\|\left(1-\theta_{m_{k}+1}\right) x+\theta_{m_{k}+1} T_{m_{k}+1} x-\left(1-\theta_{m_{k}}\right) x-\theta_{m_{k}} T_{m_{k}} x\right\| \\
& \leq\left|\theta_{m_{k}+1}-\theta_{m_{k}}\right| \sup _{x \in\left\{x_{m_{k}}\right\}}\|x\|+\theta_{m_{k}+1} \sup _{x \in\left\{x_{m_{k}}\right\}}\left\|T_{m_{k}+1} x-T_{m_{k}} x\right\| \\
& \quad+\left|\theta_{m_{k}+1}-\theta_{m_{k}}\right| \sup _{x \in\left\{x_{m_{k}}\right\}}\left\|T_{m_{k}} x\right\| \\
& \leq\left|\theta_{m_{k}+1}-\theta_{m_{k}}\right|\left(\sup _{x \in\left\{x_{m_{k}}\right\}}\|x\|+\sup _{x \in\left\{x_{m_{k}}\right\}}\left\|T_{m_{k}} x\right\|\right)+\sup _{x \in\left\{x_{m_{k}}\right\}}\left\|T_{m_{k}+1} x-T_{m_{k}} x\right\|<\infty,
\end{aligned}
$$

that is, $\left\{S_{m_{k}}\right\}_{k=1}^{\infty}$ satisfies the $A K T T$-condition. Then, by (30) and Lemma 2.10, we get that

$$
\begin{aligned}
& \left\|x_{m_{k}}-S x_{m_{k}}\right\| \\
& \quad \leq\left\|x_{m_{k}}-S_{m_{k}} x_{m_{k}}\right\|+\left\|S_{m_{k}} x_{m_{k}}-S x_{m_{k}}\right\| \\
& \quad \leq\left\|x_{m_{k}}-S_{m_{k}} x_{m_{k}}\right\|+\sup _{x \in\left\{x_{m_{k}}\right\}}\left\|S_{m_{k}} x-S x\right\| \rightarrow 0 \text { as } k \rightarrow \infty .
\end{aligned}
$$

By the same argument as in Case 1, we can show that

$$
\limsup _{k \rightarrow \infty}\left\{f\left(x^{*}\right)-x^{*}, j\left(x_{m_{k}}-x^{*}\right)\right\rangle \leq 0
$$

It follows from (31) that

$$
\begin{aligned}
\left\|S_{m_{k}} z_{m_{k}}-x_{m_{k}}\right\| & \leq\left\|S_{m_{k}} z_{m_{k}}-S_{m_{k}} x_{m_{k}}\right\|+\left\|S_{m_{k}} x_{m_{k}}-x_{m_{k}}\right\| \\
& \leq\left\|z_{m_{k}}-x_{m_{k}}\right\|+\left\|S_{m_{k}} x_{m_{k}}-x_{m_{k}}\right\| \\
& =\left(1-s_{m_{k}}\right)\left(1-\beta_{m_{k}}\right)\left\|S_{m_{k}} x_{m_{k}}-x_{m_{k}}\right\|+\left\|S_{m_{k}} x_{m_{k}}-x_{m_{k}}\right\| \\
& \leq 2\left\|x_{m_{k}}-S_{m_{k}} x_{m_{k}}\right\| \rightarrow 0 \quad \text { as } k \rightarrow \infty,
\end{aligned}
$$

and hence

$$
\begin{aligned}
\left\|x_{m_{k}+1}-x_{m_{k}}\right\| & \leq\left\|\alpha_{m_{k}}\left(f\left(x_{m_{k}}\right)-x_{m_{k}}\right)+\left(1-\alpha_{m_{k}}\right)\left(S_{m_{k}} z_{m_{k}}-x_{m_{k}}\right)\right\| \\
& \leq \alpha_{m_{k}}\left\|f\left(x_{m_{k}}\right)-x_{m_{k}}\right\|+\left(1-\alpha_{m_{k}}\right)\left\|S_{m_{k}} z_{m_{k}}-x_{m_{k}}\right\| \rightarrow 0 \quad \text { as } k \rightarrow \infty .
\end{aligned}
$$

Then, we also have

$$
\limsup _{k \rightarrow \infty}\left\langle f\left(x^{*}\right)-x^{*}, j_{q}\left(x_{m_{k}+1}-x^{*}\right)\right\rangle \leq 0 .
$$


Again from (27) we have

$$
\begin{aligned}
& \left\|x_{m_{k}+1}-x^{*}\right\|^{q} \\
& \quad \leq\left(1-(1-\rho) \alpha_{m_{k}}\right)\left\|x_{m_{k}}-x^{*}\right\|^{q}+q \alpha_{m_{k}}\left\langle f\left(x^{*}\right)-x^{*}, j_{q}\left(x_{m_{k}+1}-x^{*}\right)\right\rangle,
\end{aligned}
$$

which implies that

$$
\begin{aligned}
(1-\rho) \alpha_{m_{k}}\left\|x_{m_{k}}-x^{*}\right\|^{q} \leq & \left\|x_{m_{k}}-x^{*}\right\|^{q}-\left\|x_{m_{k}+1}-x^{*}\right\|^{q} \\
& +q \alpha_{m_{k}}\left\langle f\left(x^{*}\right)-x^{*}, j_{q}\left(x_{m_{k}+1}-x^{*}\right)\right\rangle \\
\leq & q \alpha_{m_{k}}\left\langle f\left(x^{*}\right)-x^{*}, j_{q}\left(x_{m_{k}+1}-x^{*}\right)\right\rangle .
\end{aligned}
$$

Since $\alpha_{m_{k}}>0$, we get $\lim _{k \rightarrow \infty}\left\|x_{m_{k}}-x^{*}\right\|=0$. So, we have

$$
\begin{aligned}
\left\|x_{k}-x^{*}\right\| & \leq\left\|x_{m_{k}+1}-x^{*}\right\| \\
& =\left\|x_{m_{k}}-x^{*}\right\|+\left\|x_{m_{k}+1}-x^{*}\right\|-\left\|x_{m_{k}}-x^{*}\right\| \\
& \leq\left\|x_{m_{k}}-x^{*}\right\|+\left\|x_{m_{k}+1}-x_{m_{k}}\right\| \rightarrow 0 \text { as } k \rightarrow \infty,
\end{aligned}
$$

which implies that $x_{k} \rightarrow x^{*}$ as $k \rightarrow \infty$. This completes the proof.

Applying Theorem 3.1 to a 2-uniformly smooth Banach space, we obtain the following result.

Corollary 3.2 Let $C$ be a nonempty closed convex subset of a real uniformly convex and 2-uniformly smooth Banach space $E$. Let $f \in \Pi_{C}$ with coefficient $\rho \in(0,1)$, and let $\left\{T_{n}\right\}_{n=1}^{\infty}: C \rightarrow C$ be a family of $\lambda$-strict pseudo-contractions such that $\Omega:=\bigcap_{n=1}^{\infty} F\left(T_{n}\right) \neq \emptyset$. For all $x \in C$, define the mapping $S_{n} x=(1-\theta) x+\theta T_{n} x$, where $0<\theta \leq \delta, \delta=\min \left\{1, \frac{\lambda}{K^{2}}\right\}$, and $\sum_{n=1}^{\infty}\left|\theta_{n+1}-\theta_{n}\right|<\infty$. For given $x_{1} \in C$, let $\left\{x_{n}\right\}$ be a sequence generated by

$$
\left\{\begin{array}{l}
\bar{x}_{n+1}=\beta_{n} x_{n}+\left(1-\beta_{n}\right) S_{n} x_{n}, \\
x_{n+1}=\alpha_{n} f\left(x_{n}\right)+\left(1-\alpha_{n}\right) S_{n}\left(t_{n} x_{n}+\left(1-t_{n}\right) \bar{x}_{n+1}\right), \quad n \geq 1,
\end{array}\right.
$$

where $\left\{\alpha_{n}\right\},\left\{\beta_{n}\right\}$, and $\left\{t_{n}\right\}$ are sequences in $(0,1)$ satisfying the conditions $(C 1)$ and $(C 2)$ of Theorem 3.1. Suppose in addition that $\left(\left\{T_{n}\right\}_{n=1}^{\infty}, T\right)$ satisfies the AKTT-condition. Then $\left\{x_{n}\right\}$ converges strongly to $x^{*}=Q(f) \in \Omega$, which solves the variational inequality

$$
\langle(I-f) Q(f), j(Q(f)-z)\rangle \leq 0, \quad \forall z \in \Omega,
$$

where $Q$ is a sunny nonexpansive retraction of $C$ onto $\Omega$.

Utilizing the fact that a Hilbert space $H$ is uniformly convex and 2-uniformly smooth with the best smooth constant $\kappa_{2}=1$, we obtain the following result.

Corollary 3.3 Let $C$ be a nonempty closed convex subset of a Hilbert space H. Let $f \in$ $\Pi_{C}$ with coefficient $\rho \in(0,1)$, and let $\left\{T_{n}\right\}_{n=1}^{\infty}: C \rightarrow C$ be a family of $\lambda$-strict pseudocontractions with $\lambda \in[0,1)$ such that $\Omega:=\bigcap_{n=1}^{\infty} F\left(T_{n}\right) \neq \emptyset$. For all $x \in C$, define the mapping 
$S_{n} x=\left(1-\theta_{n}\right) x+\theta_{n} T_{n} x$, where $0<\theta_{n} \leq \delta, \delta=\min \{1,2 \lambda\}$, and $\sum_{n=1}^{\infty}\left|\theta_{n+1}-\theta_{n}\right|<\infty$. For given $x_{1} \in C$, let $\left\{x_{n}\right\}$ be a sequence generated by

$$
\left\{\begin{array}{l}
\bar{x}_{n+1}=\beta_{n} x_{n}+\left(1-\beta_{n}\right) S_{n} x_{n}, \\
x_{n+1}=\alpha_{n} f\left(x_{n}\right)+\left(1-\alpha_{n}\right) S_{n}\left(t_{n} x_{n}+\left(1-t_{n}\right) \bar{x}_{n+1}\right), \quad n \geq 1,
\end{array}\right.
$$

where $\left\{\alpha_{n}\right\},\left\{\beta_{n}\right\}$, and $\left\{t_{n}\right\}$ are sequences in $(0,1)$ satisfying conditions $(C 1)$ and $(C 2)$ of Theorem 3.1. Suppose, in addition, that $\left(\left\{T_{n}\right\}_{n=1}^{\infty}, T\right)$ satisfies the AKTT-condition. Then $\left\{x_{n}\right\}$ converges strongly to $x^{*}=P(f) \in \Omega$, which solves the variational inequality

$$
\langle(I-f) P(f), P(f)-z\rangle \leq 0, \quad z \in \Omega
$$

where $P$ is a metric projection of $C$ onto $\Omega$.

\section{Application}

\subsection{The generalized viscosity explicit rules for convex combination of family of mappings}

In this subsection, we apply our main result to convex combination of a countable family of strict pseudo-contractions. The following lemmas can be found in [36, 37].

Lemma 4.1 ([36, 37]) Let $C$ be a closed convex subset of a smooth Banach space E. Suppose that $\left\{T_{n}\right\}_{n=1}^{\infty}: C \rightarrow C$ is a family of $\lambda$-strictly pseudo-contractive mappings with $\bigcap_{n=1}^{\infty} F\left(T_{n}\right) \neq \varnothing$ and $\left\{\mu_{n}\right\}_{n=1}^{\infty}$ is a real sequence in $(0,1)$ such that $\sum_{n=1}^{\infty} \mu_{n}=1$. Then the following conclusions hold:

(i) A mapping $G: C \rightarrow E$ defined by $G:=\sum_{n=1}^{\infty} \mu_{n} T_{n}$ is a $\lambda$-strictly pseudocontractive mapping.

(ii) $F(G)=\bigcap_{n=1}^{\infty} F\left(T_{n}\right)$.

Lemma 4.2 ([37]) Let $C$ be a closed convex subset of a smooth Banach space E. Suppose that $\left\{T_{k}\right\}_{k=1}^{\infty}: C \rightarrow C$ is a countable family of $\lambda$-strictly pseudocontractive mappings with $\bigcap_{k=1}^{\infty} F\left(S_{k}\right) \neq \emptyset$. For all $n \in \mathbb{N}$, define $S_{n}: C \rightarrow C$ by $S_{n} x:=\sum_{k=1}^{n} \mu_{n}^{k} T_{k} x$ for all $x \in C$, where $\left\{\mu_{n}^{k}\right\}$ is a family of nonnegative numbers satisfying the following conditions:

(i) $\sum_{k=1}^{n} \mu_{n}^{k}=1$ for all $n \in \mathbb{N}$;

(ii) $\mu^{k}:=\lim _{n \rightarrow \infty} \mu_{n}^{k}>0$ for all $k \in \mathbb{N}$;

(iii) $\sum_{n=1}^{\infty} \sum_{k=1}^{n}\left|\mu_{n+1}^{k}-\mu_{n}^{k}\right|<\infty$.

Then:

(1) Each $T_{n}$ is a $\lambda$-strictly pseudocontractive mapping.

(2) $\left\{T_{n}\right\}$ satisfies the AKTT-condition.

(3) If $T: C \rightarrow C$ is defined by $T x=\sum_{k=1}^{\infty} \mu^{k} S_{k} x$ for all $x \in C$, then, $T x=\lim _{n \rightarrow \infty} T_{n} x$ and $F(T)=\bigcap_{n=1}^{\infty} F\left(T_{n}\right)=\bigcap_{k=1}^{\infty} F\left(S_{k}\right)$.

Using Theorem 3.1 and Lemmas 4.1 and 4.2, we obtain the following result.

Theorem 4.3 Let $C$ be a nonempty closed convex subset of a real uniformly convex and q-uniformly smooth Banach space E. Let $f \in \Pi_{C}$ with coefficient $\rho \in(0,1)$, and let $\left\{T_{k}\right\}_{k=1}^{\infty}$ : $C \rightarrow C$ be a countable family of $\lambda_{k}$-strict pseudo-contractions with $\inf \left\{\lambda_{k}: k \in \mathbb{N}\right\}=\lambda>0$. 
For all $x \in C$, define a mapping $S_{n} x:=\left(1-\theta_{n}\right) x+\theta_{n} \sum_{k=1}^{n} \mu_{n}^{k} T_{k} x$ such that $\Omega:=\bigcap_{k=1}^{\infty} F\left(T_{k}\right) \neq$ $\emptyset$, where $0<\theta_{n} \leq \delta, \delta=\min \left\{1,\left(\frac{q \lambda}{\kappa_{q}}\right)^{\frac{1}{q-1}}\right\}$, and $\sum_{n=1}^{\infty}\left|\theta_{n+1}-\theta_{n}\right|<\infty$. For given $x_{1} \in C$, let $\left\{x_{n}\right\}$ be a sequence generated by

$$
\left\{\begin{array}{l}
\bar{x}_{n+1}=\beta_{n} x_{n}+\left(1-\beta_{n}\right) S_{n} x_{n}, \\
x_{n+1}=\alpha_{n} f\left(x_{n}\right)+\left(1-\alpha_{n}\right) S_{n}\left(t_{n} x_{n}+\left(1-t_{n}\right) \bar{x}_{n+1}\right), \quad n \geq 1,
\end{array}\right.
$$

where $\left\{\alpha_{n}\right\},\left\{\beta_{n}\right\}$, and $\left\{t_{n}\right\}$ are sequences in $(0,1)$ satisfy conditions $(C 1)$ and $(C 2)$ of Theorem 3.1, and $\left\{\mu_{n}^{k}\right\}$ is a real sequence satisfying (i)-(iii) of Lemma 4.2. Then $\left\{x_{n}\right\}$ converges strongly to a $x^{*} \in \Omega$.

\subsection{The generalized viscosity explicit rules for zeros of accretive operators}

In this subsection, we apply our main result to problem of finding a zero of an accretive operator. An operator $A \subset E \times E$ is said to be accretive if for all $\left(x_{1}, y_{1}\right)$ and $\left(x_{2}, y_{2}\right) \in A$, there exists $j_{q} \in J_{q}\left(x_{1}-x_{2}\right)$ such that $\left\langle y_{1}-y_{2}, j_{q}\right\rangle \geq 0$. An operator $A$ is said to satisfy the range condition if $\overline{D(A)}=R(I+\lambda A)$ for all $\lambda>0$, where $D(A)$ is the domain of $A, R(I+\lambda A)$ is the range of $I+\lambda A$, and $\overline{D(A)}$ is the closure of $D(A)$. If $A$ is an accretive operator that satisfies the range condition, then we can defined a single-valued mapping $J_{\lambda}^{A}: R(I+\lambda A) \rightarrow D(A)$ by $J_{\lambda}=(I+\lambda A)^{-1}$, which is called the resolvent of $A$. We denote $A^{-1} 0$ by the set of zeros of $A$, that is, $A^{-1} 0=\{x \in D(A): 0 \in A x\}$. It is well known that $J_{\lambda}$ is nonexpansive and $F\left(J_{\lambda}\right)=A^{-1} 0$ (see [38]). We also know the following [39]: For all $\lambda, \mu>0$ and $x \in R(I+\lambda A) \cap R(I+\mu A)$, we have

$$
\left\|J_{\lambda} x-J_{\mu} x\right\| \leq \frac{|\lambda-\mu|}{\lambda}\left\|x-J_{\lambda} x\right\| .
$$

Lemma 4.4 ([34]) Let $C$ be a nonempty closed convex subset of a Banach space E. Let $A \subset E \times E$ be an accretive operator such that $A^{-1} 0 \neq \emptyset$, which satisfies the condition $\overline{D(A)} \subset C \subset \bigcap_{\lambda>0} R(I+\lambda A)$. Suppose that $\left\{\lambda_{n}\right\} \subset(0, \infty)$ such that $\inf \left\{\lambda_{n}: n \in \mathbb{N}\right\}>0$ and $\sum_{n=1}^{\infty}\left|\theta_{n+1}-\theta_{n}\right|<\infty$. Then, $\left\{J_{\lambda_{n}}\right\}$ satisfies the AKTT-condition. Consequently, for each $x \in C,\left\{J_{\lambda_{n}} x\right\}$ converges strongly to some point of $C$. Moreover, let $J_{\lambda}: C \rightarrow C$ be defined by $J_{\lambda} x=\lim _{n \rightarrow \infty} J_{\lambda_{n}} x$ for all $x \in C$ and $F\left(J_{\lambda}\right)=\bigcap_{n=1}^{\infty} F\left(J_{\lambda_{n}}\right)$, where $\lambda_{n} \rightarrow \lambda$ as $n \rightarrow \infty$. Then, $\lim _{n \rightarrow \infty} \sup _{x \in C}\left\|J_{\lambda} x-J_{\lambda_{n}} x\right\|=0$.

Utilizing Theorem 3.1 and and Lemma 4.4, we obtain the following result.

Theorem 4.5 Let $C$ be a nonempty closed convex subset of a q-uniformly smooth Banach space E. Let $f \in \Pi_{C}$ with coefficient $\rho \in(0,1)$ and let $A \subset E \times E$ be an accretive operator such that $A^{-1} 0 \neq \emptyset$ which satisfies the condition $\overline{D(A)} \subset C \subset \bigcap_{\lambda>0} R(I+\lambda A)$. Suppose that $\left\{\lambda_{n}\right\} \subset(0, \infty)$ is such that $\inf \left\{\lambda_{n}: n \in \mathbb{N}\right\}>0$ and $\sum_{n=1}^{\infty}\left|\lambda_{n+1}-\lambda_{n}\right|<\infty$. For given $x_{1} \in C$, let $\left\{x_{n}\right\}$ be the sequence generated by

$$
\left\{\begin{array}{l}
\bar{x}_{n+1}=\beta_{n} x_{n}+\left(1-\beta_{n}\right) J_{\lambda_{n}} x_{n} \\
x_{n+1}=\alpha_{n} f\left(x_{n}\right)+\left(1-\alpha_{n}\right) J_{\lambda_{n}}\left(t_{n} x_{n}+\left(1-t_{n}\right) \bar{x}_{n+1}\right), \quad n \geq 1
\end{array}\right.
$$

where $\left\{\alpha_{n}\right\},\left\{\beta_{n}\right\}$, and $\left\{t_{n}\right\}$ are sequences in $(0,1)$ satisfying conditions $(C 1)$ and $(C 2)$ of Theorem 3.1. Then $\left\{x_{n}\right\}$ converges strongly to $x^{*} \in A^{-1} 0$. 


\subsection{The generalized viscosity explicit rules with weak contraction}

In this subsection, we apply our main result to the viscosity approximation method with weak contraction.

Definition 4.6 ([40-42]) Let $C$ be a closed and convex subset of a real Banach space $E$. A mapping $g: C \rightarrow C$ is said to be weakly contractive if there exists a continuous strictly increasing function $\psi:[0, \infty) \rightarrow[0, \infty)$ with $\psi(0)=0$ and $\lim _{t \rightarrow \infty} \psi(t)=\infty$ such that

$$
\|g(x)-g(y)\| \leq\|x-y\|-\psi(\|x-y\|), \quad x, y \in C .
$$

As a particular case, if $\psi(t)=(1-\rho) t$ for all $t \geq 0$, where $\rho \in(0,1)$, then the weakly contractive mapping is contraction with coefficient $\rho$.

In 2001, Rhoades [42] first proved Banach's contraction principle for the weakly contractive mapping in complete metric space.

Lemma 4.7 ([42]) Let $(E, d)$ be a complete metric space, and let $g$ be a weakly contractive mapping on $E$. Then $g$ has a unique fixed point in $E$.

Lemma 4.8 ([43]) Assume that $\left\{a_{n}\right\}$ and $\left\{b_{n}\right\}$ are sequences of nonnegative real number, and $\left\{\lambda_{n}\right\}$ is a sequence of a positive real number satisfying the conditions $\sum_{n=1}^{\infty} \lambda_{n}=\infty$ and $\lim _{n \rightarrow \infty} \frac{b_{n}}{\lambda_{n}}=0$. Suppose that

$$
a_{n+1} \leq a_{n}-\lambda_{n} \psi\left(a_{n}\right)+b_{n}, \quad n \geq 1,
$$

where $\psi(t)$ is a continuous strictly increasing function on $\mathbb{R}$ with $\psi(0)=0$. Then, $\lim _{n \rightarrow \infty} a_{n}=0$.

Utilizing Theorem 3.1, we obtain the following result.

Theorem 4.9 Let $C$ be a nonempty closed convex subset of a real uniformly convex and q-uniformly smooth Banach space E. Let $g: C \rightarrow C$ be a weak contraction, and let $\left\{T_{n}\right\}_{n=1}^{\infty}$ : $C \rightarrow C$ be a family of $\lambda$-strict pseudo-contractions such that $\Omega:=\bigcap_{n=1}^{\infty} F\left(T_{n}\right) \neq \emptyset$. For all $x \in C$, define the mapping $S_{n} x=\left(1-\theta_{n}\right) x+\theta_{n} T_{n} x$, where $0<\theta_{n} \leq \delta, \delta=\min \left\{1,\left(\frac{q \lambda}{\kappa_{q}}\right)^{\frac{1}{q-1}}\right\}$, and $\sum_{n=1}^{\infty}\left|\theta_{n+1}-\theta_{n}\right|<\infty$. For given $x_{1} \in C$, let $\left\{x_{n}\right\}$ be the sequence generated by

$$
\left\{\begin{array}{l}
\bar{x}_{n+1}=\beta_{n} x_{n}+\left(1-\beta_{n}\right) S_{n} x_{n}, \\
x_{n+1}=\alpha_{n} g\left(x_{n}\right)+\left(1-\alpha_{n}\right) S_{n}\left(t_{n} x_{n}+\left(1-t_{n}\right) \bar{x}_{n+1}\right), \quad n \geq 1,
\end{array}\right.
$$

where $\left\{\alpha_{n}\right\},\left\{\beta_{n}\right\}$, and $\left\{t_{n}\right\}$ are sequences in $(0,1)$ satisfy conditions $(C 1)$ and $(C 2)$ of Theorem 3.1. Suppose in addition that $\left(\left\{T_{n}\right\}_{n=1}^{\infty}, T\right)$ satisfies the AKTT-condition. Then $\left\{x_{n}\right\}$ converges strongly to $x^{*} \in \Omega$.

Proof By the smoothness of $E$ there exists a sunny nonexpansive retraction $Q$ from $C$ onto $\Omega$. Moreover, $Q(g)$ is a weakly contractive mapping of $C$ into itself. For all $x, y \in C$, we have

$$
\|Q(g(x))-Q(g(y))\| \leq\|g(x)-g(y)\| \leq\|x-y\|-\psi(\|x-y\|) .
$$


Lemma 4.7 guarantees that $Q(g)$ has a unique fixed point $x^{*} \in C$ such that $x^{*}=Q(g)$. Now, we define a sequence $\left\{y_{n}\right\}$ and $y_{1} \in C$ as follows:

$$
\left\{\begin{array}{l}
\bar{y}_{n+1}=\beta_{n} y_{n}+\left(1-\beta_{n}\right) S_{n} y_{n}, \\
y_{n+1}=\alpha_{n} g\left(y_{n}\right)+\left(1-\alpha_{n}\right) S_{n}\left(t_{n} y_{n}+\left(1-t_{n}\right) \bar{y}_{n+1}\right), \quad n \geq 1 .
\end{array}\right.
$$

Then, by Theorem 3.1 with a constant $f=g\left(x^{*}\right)$, we have that $\left\{y_{n}\right\}$ converges strongly to $\left.x^{*}=Q(g)\right) \in \Omega$. Next, we show that $x_{n} \rightarrow x^{*}$ as $n \rightarrow \infty$. Since

$$
\left\|\bar{x}_{n+1}-\bar{y}_{n+1}\right\| \leq \beta_{n}\left\|x_{n}-y_{n}\right\|+\left(1-\beta_{n}\right)\left\|S_{n} x_{n}-S_{n} y_{n}\right\| \leq\left\|x_{n}-y_{n}\right\|,
$$

it follows that

$$
\begin{aligned}
&\left\|x_{n+1}-y_{n+1}\right\| \\
&=\left\|\alpha_{n}\left(g\left(x_{n}\right)-g\left(x^{*}\right)\right)+\left(1-\alpha_{n}\right)\left(S_{n}\left(t_{n} x_{n}+\left(1-t_{n}\right) \bar{x}_{n+1}\right)-S_{n}\left(t_{n} y_{n}+\left(1-t_{n}\right) \bar{y}_{n+1}\right)\right)\right\| \\
& \leq \alpha_{n}\left\|g\left(x_{n}\right)-g\left(x^{*}\right)\right\|+\left(1-\alpha_{n}\right)\left\|S_{n}\left(t_{n} x_{n}+\left(1-t_{n}\right) \bar{x}_{n+1}\right)-S_{n}\left(t_{n} y_{n}+\left(1-t_{n}\right) \bar{y}_{n+1}\right)\right\| \\
& \leq \alpha_{n}\left\|g\left(x_{n}\right)-g\left(y_{n}\right)\right\|+\alpha_{n}\left\|g\left(y_{n}\right)-g\left(x^{*}\right)\right\| \\
& \quad+\left(1-\alpha_{n}\right)\left(t_{n}\left\|x_{n}-y_{n}\right\|+\left(1-t_{n}\right)\left\|\bar{x}_{n+1}-\bar{y}_{n+1}\right\|\right) \\
& \leq \alpha_{n}\left\|x_{n}-y_{n}\right\|-\alpha_{n} \psi\left(\left\|x_{n}-y_{n}\right\|\right)+\alpha_{n}\left\|y_{n}-x^{*}\right\| \\
& \quad-\alpha_{n} \psi\left(\left\|y_{n}-x^{*}\right\|\right)+\left(1-\alpha_{n}\right)\left\|x_{n}-y_{n}\right\| \\
& \leq\left\|x_{n}-y_{n}\right\|-\alpha_{n} \psi\left(\left\|x_{n}-y_{n}\right\|\right)+\alpha_{n}\left\|y_{n}-x^{*}\right\| .
\end{aligned}
$$

Since $\left\{y_{n}\right\}$ converges strongly to $x^{*}$, applying Lemma 4.8 to (43), we obtain that $\lim _{n \rightarrow \infty}\left\|x_{n}-y_{n}\right\|=0$. Therefore $x_{n} \rightarrow x^{*}$. This completes the proof.

\section{Numerical examples}

In this section, we present a numerical example of our main result.

Example 5.1 Let $E=\ell_{4}$ and $C=\left\{\mathbf{x}=\left(x_{1}, x_{2}, x_{3}, x_{4}, \ldots\right) \in \ell_{4}: x_{i} \in \mathbb{R}\right.$ for $\left.i=1,2,3, \ldots\right\}$ with norm $\|\mathbf{x}\|_{\ell_{4}}=\left(\sum_{i=1}^{\infty}\left|x_{i}\right|^{4}\right)^{1 / 4}$. Let $f: C \rightarrow C$ be the contraction defined by $f(\mathbf{x})=\frac{1}{3} \mathbf{x}$. Let $\left\{T_{n}\right\}_{n=1}^{\infty}: C \rightarrow C$ be the strictly pseudo-contractive mapping defined by

$$
T_{n} \mathbf{x}= \begin{cases}\frac{1}{n}\left(1, \frac{1}{2}, \frac{1}{3}, \frac{1}{4}, 0,0,0, \ldots\right)-2 \mathbf{x} & \text { if } \mathbf{x} \neq \mathbf{0} \\ \mathbf{0} & \text { if } \mathbf{x}=\mathbf{0}\end{cases}
$$

where $\mathbf{0}=(0,0,0,0,0,0,0, \ldots)$ is the null vector on $\ell_{4}$.

- We show that $T_{n}$ is strictly pseudo-contractive. For each $n \geq 1$, if $\mathbf{x}, \mathbf{y} \neq \mathbf{0}$, then

$$
\begin{aligned}
\left\langle\left(I-T_{n}\right) \mathbf{x}-\left(I-T_{n}\right) \mathbf{y}, j_{2}(\mathbf{x}-\mathbf{y})\right\rangle & =\left\langle 3 \mathbf{x}-3 \mathbf{y}, j_{2}(\mathbf{x}-\mathbf{y})\right\rangle \\
& =3\|\mathbf{x}-\mathbf{y}\|_{\ell_{4}}^{2} \\
& =\frac{1}{3}\|3 \mathbf{x}-3 \mathbf{y}\|_{\ell_{4}}^{2} \\
& \geq \lambda\left\|\left(I-T_{n}\right) \mathbf{x}-\left(I-T_{n}\right) \mathbf{y}\right\|_{\ell_{4}}^{2}
\end{aligned}
$$


for $\lambda \leq \frac{1}{3}$. Then, we can choose $\lambda=\frac{1}{3}$. Thus, $T_{n}$ is $\frac{1}{3}$-strictly pseudo-contractive with $\bigcap_{n=1}^{\infty} F\left(T_{n}\right)=\{\mathbf{0}\}$. Further, we observe that $T_{n}$ is not nonexpansive.

- We show that $\left(\left\{T_{n}\right\}_{n=1}^{\infty}, T\right)$ satisfies the AKTT-condition. Since

$$
\begin{aligned}
\sup _{\mathbf{x} \in \ell_{4}} & \left\|T_{n+1} \mathbf{x}-T_{n} \mathbf{x}\right\|_{\ell_{4}} \\
& =\sup _{\mathbf{x} \in \ell_{4}}\left\|\frac{1}{n+1}\left(1, \frac{1}{2}, \frac{1}{3}, \frac{1}{4}, 0,0,0, \ldots\right)-2 \mathbf{x}-\frac{1}{n}\left(1, \frac{1}{2}, \frac{1}{3}, \frac{1}{4}, 0,0,0, \ldots\right)+2 \mathbf{x}\right\|_{\ell_{4}} \\
& =\left\|\frac{1}{n+1}\left(1, \frac{1}{2}, \frac{1}{3}, \frac{1}{4}, 0,0,0, \ldots\right)-\frac{1}{n}\left(1, \frac{1}{2}, \frac{1}{3}, \frac{1}{4}, 0,0,0, \ldots\right)\right\|_{\ell_{4}} \\
& =\left(\frac{1}{n}-\frac{1}{n+1}\right)\left\|\left(1, \frac{1}{2}, \frac{1}{3}, \frac{1}{4}, 0,0,0, \ldots\right)\right\|_{\ell_{4}} .
\end{aligned}
$$

So we have

$$
\begin{aligned}
\sum_{n=1}^{\infty} \sup _{\mathbf{x} \in \ell_{4}}\left\|T_{n+1} \mathbf{x}-T_{n} \mathbf{x}\right\|_{\ell_{4}} & =\lim _{n \rightarrow \infty} \sum_{k=1}^{n} \sup _{\mathbf{x} \in \ell_{4}}\left\|T_{k+1} \mathbf{x}-T_{k} \mathbf{x}\right\|_{\ell_{4}} \\
& =\left\|\left(1, \frac{1}{2}, \frac{1}{3}, \frac{1}{4}, 0,0,0, \ldots\right)\right\|_{\ell_{4}}<\infty,
\end{aligned}
$$

that is, $\left(\left\{T_{n}\right\}_{n=1}^{\infty}, T\right)$ satisfies the AKTT-condition, where $T: C \rightarrow C$ is defined by

$$
T \mathbf{x}=\lim _{n \rightarrow \infty} T_{n} \mathbf{x}=-2 \mathbf{x}, \quad \mathbf{x} \in C .
$$

Since in $\ell_{4}, q=2$ and $\kappa_{2}=3$, we can choose $\theta_{n}=\frac{1}{9 n}+\frac{1}{9}$. Define the mapping $\left\{S_{n}\right\}_{n=1}^{\infty}: C \rightarrow C$ by

$$
S_{n} \mathbf{x}= \begin{cases}\left(\frac{2}{3}-\frac{1}{3 n}\right) \mathbf{x}+\left(\frac{1}{9 n^{2}}+\frac{1}{9 n}\right)\left(1, \frac{1}{2}, \frac{1}{3}, \frac{1}{4}, 0,0,0, \ldots\right) & \text { if } \mathbf{x} \neq \mathbf{0} \\ \mathbf{0} & \text { if } \mathbf{x}=\mathbf{0} .\end{cases}
$$

Since $\left(\left\{T_{n}\right\}_{n=1}^{\infty}, T\right)$ satisfies the AKTT condition, we also have that $\left(\left\{S_{n}\right\}_{n=1}^{\infty}, S\right)$ satisfies the AKTT condition, where $S: C \rightarrow C$ is defined by

$$
S \mathbf{x}=\lim _{n \rightarrow \infty} S_{n} \mathbf{x}=\frac{2}{3} \mathbf{x}, \quad \mathbf{x} \in C
$$

Then, we have $F(S)=F(T)=\bigcap_{n=1}^{\infty} F\left(T_{n}\right)=\{\mathbf{0}\}$. Let $\alpha_{n}=\frac{1}{32 n+1}, \beta_{n}=\frac{1}{100 n+3}+0.32$, and $t_{n}=$ $\frac{n}{2 n+1}$. So our algorithm (16) has the following form:

$$
\left\{\begin{array}{l}
\overline{\mathbf{x}}_{n+1}=\left(\frac{1}{100 n+3}+0.32\right) \mathbf{x}_{n}+\left(0.68-\frac{1}{100 n+3}\right) S_{n} \mathbf{x}_{n}, \\
\mathbf{x}_{n+1}=\frac{1}{32 n+2} f\left(\mathbf{x}_{n}\right)+\frac{32 n}{32 n+1} S_{n}\left(\frac{n}{2 n+1} \mathbf{x}_{n}+\frac{n+1}{2 n+1} \overline{\mathbf{x}}_{n+1}\right), \quad n \geq 1 .
\end{array}\right.
$$

Let $\mathbf{x}_{1}=(1,-0.25,1.46,1.85,0,0,0, \ldots)$ be the initial point. Then, we obtain numerical results in Table 1 and Fig. 1. 
Table 1 The values of the sequences $\left\{\mathbf{x}_{n}\right\}$

\begin{tabular}{rll}
\hline$n$ & $\mathbf{x}_{n}$ & $\left\|\mathbf{x}_{n+1}-\mathbf{x}_{n}\right\|_{\ell_{4}}$ \\
\hline 1 & $(1.000000,-0.250000,1.460000,1.850000,0,0,0, \ldots)$ & $1.459 \mathrm{e}+00$ \\
50 & $(0.007006,0.003503,0.002335,0.001751,0,0,0, \ldots)$ & $1.471 \mathrm{e}-04$ \\
100 & $(0.003416,0.001708,0.001139,0.000854,0,0,0, \ldots)$ & $3.531 \mathrm{e}-05$ \\
150 & $(0.002258,0.001129,0.000753,0.000565,0,0,0, \ldots)$ & $1.549 \mathrm{e}-05$ \\
200 & $(0.001687,0.000843,0.000562,0.000422,0,0,0, \ldots)$ & $8.657 \mathrm{e}-06$ \\
$\vdots$ & $\vdots$ & $\vdots$ \\
400 & $(0.000838,0.000419,0.000279,0.000210,0,0,0, \ldots)$ & $2.143 \mathrm{e}-06$ \\
450 & $(0.000745,0.000372,0.000248,0.000186,0,0,0, \ldots)$ & $1.692 \mathrm{e}-06$ \\
500 & $(0.000670,0.000335,0.000223,0.000167,0,0,0, \ldots)$ & $1.369 \mathrm{e}-06$ \\
\hline
\end{tabular}

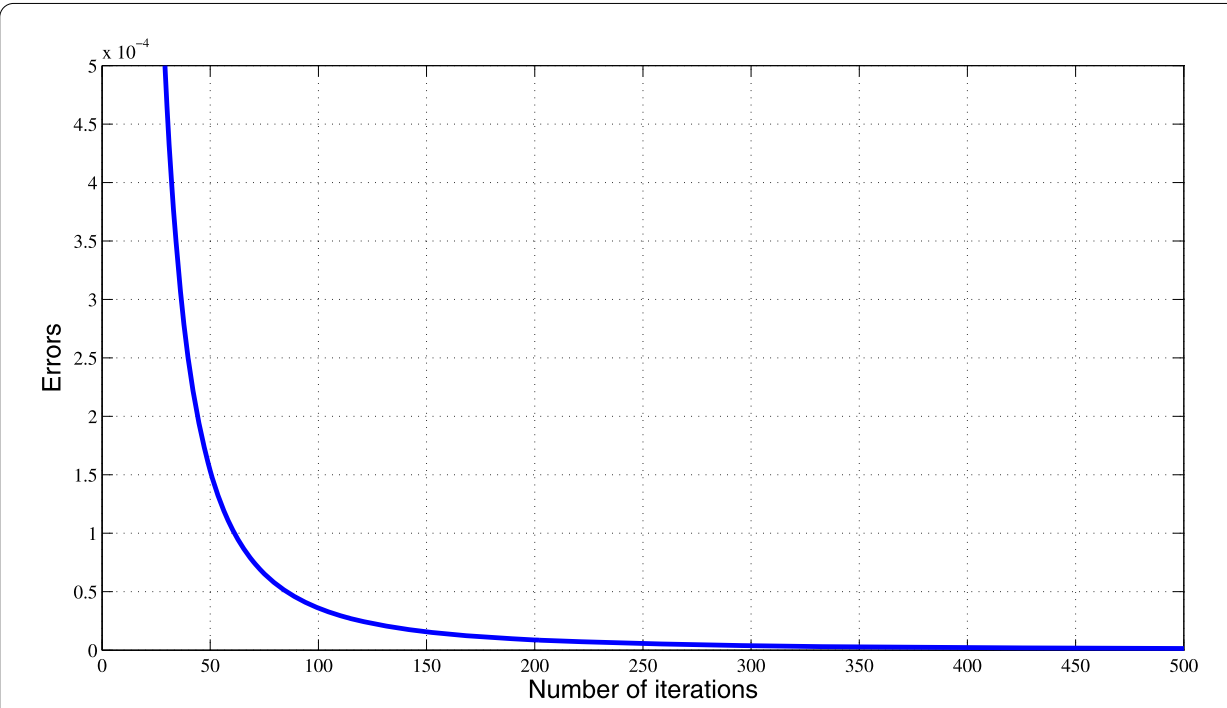

Figure 1 The behavior of errors

\section{Conclusion}

In this work, we introduce an algorithm by a generalized viscosity explicit rule for finding a common fixed point of a countable family of strictly pseudo-contractive mappings in a $q$-uniformly smooth Banach space. We obtain some strong convergence theorem for the sequence generated by the proposed algorithm under suitable conditions. However, we should like remark the following:

(1) We extend the results of Ke and Ma [21] and Marino et al. [25] from a one nonexpansive mapping in Hilbert spaces to a countable family of strictly pseudo-contractive mappings in a $q$-uniformly smooth Banach space.

(2) Our result is proved with a new assumption on the control conditions $\left\{\beta_{n}\right\}$ and $\left\{t_{n}\right\}$.

(3) The method of proof of our result is simpler in comparison with the results of [19, $21,44,45])$. Moreover, we remove the conditions $\sum_{n=1}^{\infty}\left|\alpha_{n+1}-\alpha_{n}\right|<\infty$ and $0<\epsilon \leq s_{n} \leq s_{n+1}<1$ in Theorem 3.1 of [21].

(4) We give a numerical example that shows the efficiency and implementation of our main result in the space $\ell_{4}$, which is a uniformly convex and 2-uniformly smooth Banach space but not a Hilbert space. 


\section{Acknowledgements}

The authors would like to thank the Rajamangala University of Technology Thanyaburi for financial support.

\section{Funding}

P. Sunthrayuth was supported by RMUTT research foundation scholarship of the Rajamangala University of Technology Thanyaburi under Grant No. NRF04066005.

\section{Competing interests}

The authors declare that they have no competing interests.

\section{Authors' contributions}

Both authors contributed equally to the writing of this paper. Both authors read and approved the final manuscript.

\section{Publisher's Note}

Springer Nature remains neutral with regard to jurisdictional claims in published maps and institutional affiliations.

Received: 18 February 2018 Accepted: 29 May 2018 Published online: 11 July 2018

\section{References}

1. Takahashi, W.: Nonlinear Functional Analysis. Yokohama Publishers, Yokohama (2000)

2. Browder, F.E., Petryshyn, W.V.: Construction of fixed points nonlinear mappings in Hilbert space. J. Math. Anal. Appl. 20, 197-228 (1967)

3. Scherzer, O.: Convergence criteria of iterative methods based on Landweber iteration for solving nonlinear problems. J. Math. Anal. Appl. 194, 911-933 (1991)

4. Cai, G.: Viscosity iterative algorithm for variational inequality problems and fixed point problems in a real $q$-uniformly smooth Banach space. Fixed Point Theory Appl. 2015, 67 (2015)

5. Zhang, H., Su, Y: Convergence theorems for strict pseudo-contractions in q-uniformly smooth Banach spaces. Nonlinear Anal. 71, 4572-4580 (2009)

6. Zhou, H.: Convergence theorems of fixed points for $\kappa$-strict pseudo-contractions in Hilbert spaces. Nonlinear Anal. $69,456-462(2008)$

7. Jung, J.S.: Strong convergence of iterative methods for $\kappa$-strictly pseudo-contractive mappings in Hilbert spaces. Appl. Math. Comput. 215, 3746-3753 (2010)

8. Sahu, D.R., Petruşel, A.: Strong convergence of iterative methods by strictly pseudocontractive mappings in Banach spaces. Nonlinear Anal. 74, 6012-6023 (2011)

9. Cholamjiak, P., Suantai, S.: Strong convergence for a countable family of strict pseudocontractions in $q$-uniformly smooth Banach spaces. Comput. Math. Appl. 62, 787-796 (2011)

10. Auzinger, W., Frank, R.: Asymptotic error expansions for stiff equations: an analysis for the implicit midpoint and trapezoidal rules in the strongly stiff case. Numer. Math. 56, 469-499 (1989)

11. Bader, G., Deufhard, P.: A semi-implicit mid-point rule for stiff systems of ordinary differential equations. Numer. Math. 41, 373-398 (1983)

12. Deufhard, P.: Recent progress in extrapolation methods for ordinary differential equations. SIAM Rev. 27(4), 505-535 (1985)

13. Schneider, C.: Analysis of the linearly implicit mid-point rule for differential-algebra equations. Electron. Trans. Numer. Anal. 1, 1-10 (1993)

14. Somalia, S.: Implicit midpoint rule to the nonlinear degenerate boundary value problems. Int. J. Comput. Math. 79(3), 327-332 (2002)

15. van Veldhuxzen, M.: Asymptotic expansions of the global error for the implicit midpoint rule (stiff case). Computing 33, 185-192 (1984)

16. Schneider, C.: Analysis of the linearly implicit mid-point rule for differential-algebraic equations. Electron. Trans. Numer. Anal. 1, 1-10 (1993)

17. Hairer, E., Nørsett, S.P., Wanner, G.: Solving Ordinary Differential Equations I: Nonstiff Problems, 2nd edn. Springer Series in Computational Mathematics. Springer, Berlin (1993)

18. Alghamdi, M.A., Alghamdi, M.A., Shahzad, N., Xu, H.-K.: The implicit midpoint rule for nonexpansive mappings. Fixed Point Theory Appl. 2014, 96 (2014)

19. Xu, H.-K., Alghamdi, M.A., Shahzad, N.: The viscosity technique for the implicit midpoint rule of nonexpansive mappings in Hilbert spaces. Fixed Point Theory Appl. 2015, 41 (2015)

20. Moudafi, A.: Viscosity approximation methods for fixed point problems. J. Math. Anal. Appl. 241, 46-55 (2000)

21. Ke, Y., Ma, C.: The generalized viscosity implicit rules of nonexpansive mappings in Hilbert spaces. Fixed Point Theory Appl. 2015, 190 (2015)

22. Palais, R.S., Palais, R.A.: Differential Equations, Mechanics, and Computation. American Mathematical Soc., Providence (2009)

23. Hoffman, J.D.: Numerical Methods for Engineers and Scientists, 2nd edn. Dekker, New York (2001)

24. Moaveni, S.: Finite Element Analysis Theory and Application with ANSYS, 3 edn. Pearson Education, Upper Saddle River (2008)

25. Marino, G., Scardamaglia, B., Zaccone, R.: A general viscosity explicit midpoint rule for quasi-nonexpansive mappings. J. Nonlinear Convex Anal. 18(1), 137-148 (2017)

26. Xu, Z.B., Roach, G.F.: Characteristic inequalities of uniformly smooth Banach spaces. J. Math. Anal. Appl. 157, 189-210 (1991)

27. Reich, S.: Strong convergence theorems for resolvents of accretive operators in Banach spaces. J. Math. Anal. Appl. 75 , 287-292 (1980) 
28. Song, Y., Ceng, L.: A general iteration scheme for variational inequality problem and common fixed point problems of nonexpansive mappings in q-uniformly smooth Banach spaces. J. Glob. Optim. 57, 1327-1348 (2013)

29. Cai, G., Bu, S.: An iterative algorithm for a general system of variational inequalities and fixed point problems in q-uniformly smooth Banach spaces. Optim. Lett. 7, 267-287 (2013)

30. Zhang, H., Su, Y.: Convergence theorems for strict pseudo-contractions in q-uniformly smooth Banach spaces. Nonlinear Anal. 71, 4572-4580 (2009)

31. Chidume, C.: Geometric Properties of Banach Spaces and Nonlinear Iterations. Springer, Berlin (2009)

32. Xu, H.K.: In: Inequalities in Banach Spaces with Applications, Nonlinear Analysis: Theory, Methods \& Applications, vol. 16, pp. 1127-1138 (1991)

33. Mitrinović, D.S.: Analytic Inequalities. Springer, New York (1970)

34. Aoyama, K., Kimura, Y., Takahashi, W., Toyoda, M.: Approximation of common fixed points of a countable family of nonexpansive mapping in a Banach space. Nonlinear Anal. 67, 2350-2360 (2007)

35. Maingé, P.E.: Strong convergence of projected subgradient methods for nonsmooth and nonstrictly convex minimization. Set-Valued Anal. 16, 899-912 (2008)

36. Boonchari, D., Saejung, S.: Weak and strong convergence theorems of an implicit iteration for a countable family of continuous pseudocontractive mappings. J. Comput. Appl. Math. 233, 1108-1116 (2009)

37. Boonchari, D., Saejung, S.: Construction of common fixed points of a countable family of $\lambda$-demicontractive mappings in arbitrary Banach spaces. Appl. Math. Comput. 216, 173-178 (2010)

38. Takahashi, W.: Nonlinear Functional Analysis. Yokohama Publishers, Yokohama (2000)

39. Eshita, K., Takahashi, W.: Approximating zero points of accretive operators in general Banach spaces. JP J. Fixed Point Theory Appl. 2, 105-116 (2007)

40. Alber, Ya.l., Guerre-Delabriere, S.: Principle of weakly contractive maps in Hilbert spaces. Oper. Theory, Adv. Appl. 98, 7-22 (1997)

41. Alber, Ya.l., Guerre-Delabriere, S., Zelenko, L.: Principle of weakly contractive maps in metric spaces. Commun. Appl. Nonlinear Anal. 5(1), 45-68 (1998)

42. Rhoades, B.E.: Some theorems on weakly contractive maps. Nonlinear Anal. 47, 2683-2693 (2001)

43. Alber, Ya.l., lusem, A.N.: Extension of subgradient techniques for nonsmooth optimization in Banach spaces. Set-Valued Anal. 9, 315-335 (2001)

44. Yao, Y., Shahzad, N., Liou, Y.-C.: Modified semi-implicit midpoint rule for nonexpansive mappings. Fixed Point Theory Appl. 2015, 166 (2015)

45. Luo, P., Cai, G., Shehu, Y.: The viscosity iterative algorithms for the implicit midpoint rule of nonexpansive mappings in uniformly smooth Banach spaces. J. Inequal. Appl. 2017, 154 (2017)

\section{Submit your manuscript to a SpringerOpen ${ }^{\odot}$ journal and benefit from:}

- Convenient online submission

- Rigorous peer review

- Open access: articles freely available online

- High visibility within the field

- Retaining the copyright to your article

Submit your next manuscript at $\gg$ springeropen.com 